THE PROBLEM OF how proteins are translocated across biological membranes is a salient feature in biochemistry and cell biology. In the case of the bacterial plasma membrane, the endoplasmic reticulum (ER), chloroplasts and mitochondria, many of the components involved in mediating protein translocation have been identified. However, many open questions exist with regard to several aspects of these translocation events. For example, proteins appear not to traverse membranes in a folded state, but rather as extended polypeptide chains. How is this unfolding achieved? How is energy harnessed to cause unfolding? How is the unfolded state maintained? On completion of translocation how does the protein then refold to its native structure? Another important question concerns the nature of the driving force for the movement of proteins across membranes. The translocation process is generally observed to be unidirectional; what is the basis of this phenomenon? Some insights into these questions have recently been gained. Increasing evidence indicates that the translocation process is integrally coupled to the action of a set of molecular chaperones located in the cytosol and in the mitochondrial matrix (Table l).

Mitochondria represent an attractive system in which to study the basic mechanisms and energetics of protein translocation events, for a number of reasons. First, powerful in vitro protein import systems have been established in which the pathway of protein translocation can be experimentally dissected into a series of distinct steps. Second, the bioenergetics are well documented and can be easily manipulated, enabling independent modulation of both membrane potential and levels of ATP in the matrix. Furthermore, many mutants defective in mitochondrial function have been identified, particularly in yeast. These mutants have not only resulted in the cloning of genes encoding proteins essential for mitochondrial function, but have also proved invaluable for analysis of the import process.

With the exception of a small percentage of proteins that are encoded by

R. A. Stuart, D. M. Cyr and W. Neupert are at the Institut für Physiologische Chemie der Universität München, Goethestrasse 33, 80336 München, Germany; and E. A. Graig is at the Department of Biomolecular Chemistry, University of Wisconsin-Madison, Madison, WI 53706, USA.

\title{
Mitochondrial molecular chaperones: their role in protein translocation
}

\section{Rosemary A. Stuart, Douglas M. Cyr, Elizabeth A. Craig and Walter Neupert}

After synthesis in the cytosol, most mitochondrial proteins must traverse mitochondrial membranes to reach their functional location. During this process, proteins become unfolded and then refold to attain their native conformation after crossing the lipid bilayers. Mitochondrial molecular chaperones play an essential mechanistic role at various steps of this process. They facilitate presequence translocation, unfolding of the cytosol-localized domains of precursor proteins, movement across the mitochondrial membranes and, finally, folding of newly imported proteins within the matrix. the mitochondrial genome, the majority of proteins are syrthesized in the cytoplasm as precursor proteins with amino-terminal presequences, which are proteolytically removed in the mitochondrial matrix. The precursor proteins are imported along a multistep pathway, and this process normally occurs by a post-translational mechanism (for a recent review see Ref. 1). Precursors are initially recognized by, and bind to, receptor proteins on the outer surface of mitochondria. The passage of preproteins across the inner membrane (M) is dependent on both a membrane potential, $\Delta \Psi$, across the IM, and ATP hydrolysis in the matrix. It requires the participation of a proteinaceous machinery localized in the $\mathrm{IM}$, the composition of which has only recently begun to be unravelled. In addition, the mitochondrial chaperone Hsp70 (mt-Hsp70), encoded by the SSCI gene in Saccharomyces cerevisiae ${ }^{2}$, appears to play a decisive role in the translocation of preproteins across the mitochondrial membranes. Incoming polypeptide chains are thought to represent a substrate for the matrix-localized mt-Hsp70, and, through a series of binding and release cycles, mt-Hsp70 mediates the passage of the protein into the matrix $x^{3,4}$.

In this review, we will summarize the progress made over the past year or so that has increased our understanding of the mechanism of protein translocation into mitochondria. New observations have, in particular, shed light on the importance of molecular chaperones,
Table I. Molecular chaperores !mpilicated in mitochondrlal proteln translocation altu folding in yeast

\begin{tabular}{|c|c|c|c|}
\hline Chaperone & Cellular location & Essential & $\operatorname{Ref}(\mathbf{s})$ \\
\hline SSA1/SSA2 & Cytosol & Yes & 16 \\
\hline YDJ1 & Cytosol & $\mathrm{No}^{\mathrm{a}}$ & 17,18 \\
\hline SSC1 (mt-Hsp70) & Mitochondrial matrin & Yes & 2,3 \\
\hline MDJ1 & Mitochondrial matrix & $\mathrm{No}^{\mathrm{b}}$ & c \\
\hline MGE1 & Mitochondrial matrix & Yes & d \\
\hline Hsp60 & Mitochondrial matrix & Yes & 25,26 \\
\hline Hsp10 & Mitochondrial matrix & Yes & $28, e$ \\
\hline \multicolumn{4}{|c|}{$\begin{array}{l}\text { Viable at } 23^{\circ} \mathrm{C} \text { but not at } 37^{\circ} \mathrm{C} \text {. } \\
\text { bNonvlable on nonfermentable carbon sources; viable at } 23^{\circ} \mathrm{C} \text { but not at } 37^{\circ} \mathrm{C} \text { on fermentable carbon } \\
\text { sources. } \\
\text { 'N. Rowley et al., pers. commun. } \\
\text { dS. Laloraya, B. D. Gambill and E. A. Craig, unpublished. } \\
\text { eJ. Höhfeld and F-U. Hartl, pers. commun. }\end{array}$} \\
\hline
\end{tabular}




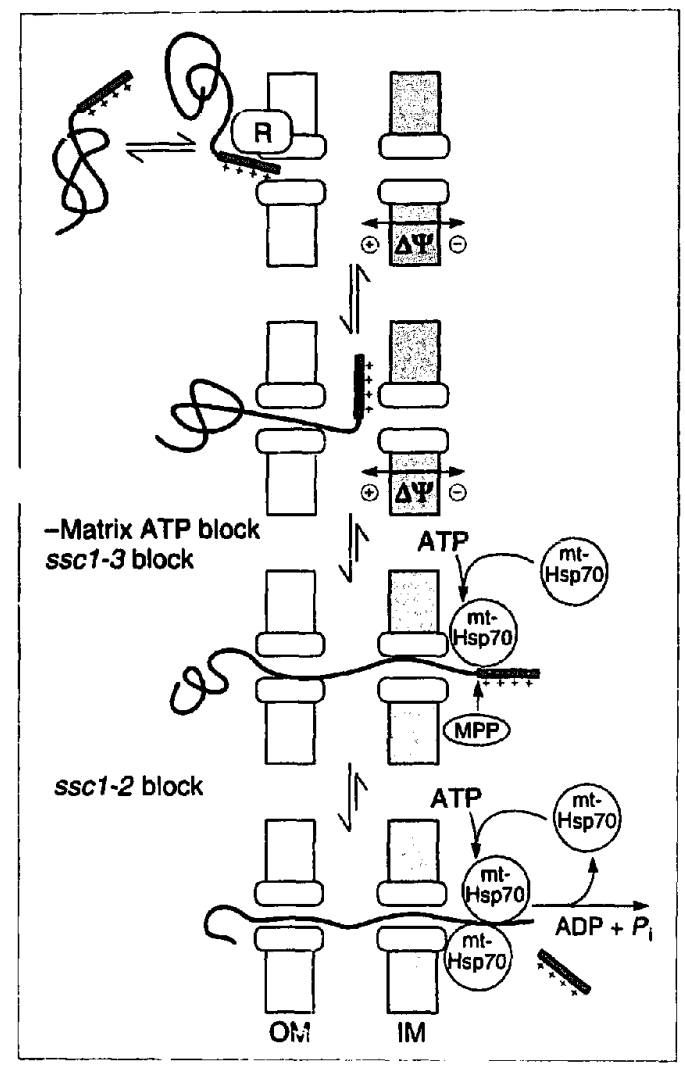

Figure 1

Model for the role of $\Delta \Psi$, matrix ATP and $\mathrm{mt}$-Hsp7O in the translocation of the presequence into the mitochondrial matrix. The positive charges and shaded box on the precursor denote the presequence, which undergoes cleavage by the matrix processing peptidase (MPP). Import steps proposed to be inhibited in the ssc1-2, ssc1.3 and matrix-ATP.depleted mitochondria are indicated, $R$, receptor; OM outer membrane, IM, inner membrane.

which appear to function at several distinct steps of the import pathway.

\section{Manipulation of mt-Hsp70 action}

Two important tools exist for studying the actions of mitochondrial chaperones, in particular mt-Hsp70; these are (1) modulation of matrix ATP concentrations to levels that adversely affect the ATP-dependent action of mt-Hsp70, and (2) mutations in the $\mathrm{SSCl}$ gene resulting in altered $\mathrm{mt}-\mathrm{Hsp} 70$ proteins that are temperature sensitive for function.

In addition to $\Delta \Psi$, hydrolysis of matrix ATP constitutes (at least part of) the driving force for the vectorial movement of preproleins into the matrix. A critical role of matrix ATP in protein import appears to be through its facilitating the reversible binding of $\mathrm{mt}$ Hsp70 to incoming polypeptides. In isolated mitochondria, matrix ATP can be stringently reduced to experimentally definable levels; thus it is possible to directly address which specific stages of import are affected when ATP levels become limiting for mtHsp70 function ${ }^{5-8}$. Other ATP-requiring chaperones, such as Hsp60, do not play a role in translocation per se, and hence are not determining factors in these energetic studies.

Temperature-sensitive (ts) mutants of the $\mathrm{SSC}$ 1 gene have also proved invaluable for the study of $\mathrm{mt}-\mathrm{Hsp} 70$ functions. In addition to the previously published $s s c 1-2$ allele, a second ts allele of the Hsp70 gene, ssc1-3, was recently identified. Import of preproteins into mitochondria was inhibited both in vitro and in vivo in $\operatorname{sscl-2}$ at nonpermissive temperatures ${ }^{9}$. In ssc $1-3$ mitochondria, mt-Hsp70 function was more severeiy affected than in the sscl-2 mutant (see below). The sscl-3 mutation mapped to the amino-terminal AlTPase domaln, in contrast with the sscl.2 mutation, which was localized to the putative peptide-binding domain at the carboxyterminal portion of mt-Hsp70. Mt-Hsp70 from the ssc1-2 mutant displays the ability to bind substrates (release being proposed to be affected), whereas it was suggested that the function of $\mathrm{mt}-\mathrm{Hsp} 70$ in the ssc1.3 mutant was compromised at the initial level of binding to substrates?

Comparison of these two mutants, together with the studies of ATP-depleted mitochondria, has revealed new insights into the role of mt-Hsp70, and, more specifically, has implicated the direct involvement of $\mathrm{mt}-\mathrm{Hsp} 70$ in three distinct siajos of precursor protein insport: (1) binding to amino-terminal regions of the incoming polypeptide in order to stabilize the targetting presequence on the trans-side of the IM, thereby making the initial import step irreversible; (2) unfolding of tightly folded segments of preproteins on the cis-side of the outer membrane (OM), facilitated by binding of mt-Hsp70 to the matrix-exposed domains of the polypeptide; and (3) completion of translocation across the IM by binding to further domains of the preprotein.

In the following sections, we summarize the findings that gave rise to these conclusions, and present a model for the action of mt-Hsp70 that includes the involvement of other recently identified mitochondrial chaperones.

\section{Matrix ATP and mt-Hsp70 are required for translocation of the presequence across the III}

The passage of the targetting presequence across the $I M$ in response to $\Delta \Psi$ represents the first committed step of translocation across the IM. It has recently become clear that mt-Hsp70 plays an important role in this early step of import, trapping the presequence in the matrix following its initial translocation triggered by $\Delta \Psi$ (Fig. 1).

Drastic reduction of matrix ATP to low micromolar levels caused not only complete inhibition of import, but also of presequence translocation across the $\mathrm{IM}$ and subsequent processing in the matrix, despite the presence of $\Delta \Psi$. Thus, in the absence of $\mathrm{mt}-\mathrm{Hsp} 70$ function, the precursors accumulated as import intermediates, being both unprocessed by matrix processing peptidase (MPP) and accessible to exogenously added proteases. These import intermedlates were arrested at an early stage of import, since their further chase required not only the presence of ATP in the matrix, but also the $\Delta \Psi$ across the $\mathrm{IM}^{7,10}$. Similar accumulation of unprocessed external import intermediates was observed (with the same precursors) in the sscl-3 mutant (D. M. Cyr, R. A. Stuart and W. Neupert, unpublished).

Presequence translocation across the IM therefore appears to occur in response to $\Delta \Psi$. However, it seems to be a reversible process (Fig. 1). Mt-Hsp70, by binding to the incoming polypeptides upon their exposure to the matrix, makes this import event unidirectional by preventing the polypeptide from slipping back to the cis-side of the IM. Binding of mt-Hsp70 could occur through direct interaction of mt-Hsp70 with the presequence, or with aminoterminal mature regions of the precursor. It appears that the mt-Hsp70 is competent for binding substrates in the matrix when it is in its ATP-bound state, and is thus compromised under conditions of matrix-ATP depletion. The 
extent to which mt-Hsp70-ATP is required for presequence presentation to MPP was observed to vary from precursor to precursor and most probably reflects the stability and depth to which the presequence can be initially inserted into the IM.

\section{Binding of $\mathrm{mt}$-Hsp70 to incoming polypeptides is required for completion of translocation}

Completion of import, in contrast with translocation of the presequence, does not require $\Delta \Psi$, but does require matrix ATP hydrolysis. This requirement for ATP is thought to reflect the ATP-dependent action of mt-Hsp70, a notion that is supported by the characterization of the ssc1-2 ts mutant, in which unprocessed precursors accumulated in vivo when yeast cells were exposed to nonpermissive temperatures.

When mitochondria from the sscl-2 mutant were incubated at the nonpermissive temperature, both insertion and processing of preproteins could occur. However, these precursors remained accessible to exogenously added proteases. Thus, in the ssc 1.2 mutant under conditions where mtHsp70 action was impaired, preproteins accumulated as intermediates spanning both mitochondrial membranes, agrin suggesting that mt-Hsp70 acts at an zarly step in the translocation process. Interestingly, such spanning intermediates could be both co-immunoprecipitated and crosslinked with mt-Hsp70, indicating that mt-Hsp70 directly interacts with these precursors during the translocation process ${ }^{3,11}$.

By contrast, in vitro import in the sscl-3 ts mutant was more drastically affected than in the ssc1-2 mutant. Both import into the matrix and precursor processing were inhibited in mitochondria from this mutant at nonpermissive temperatures. These unprocessed precursors accumulated on the outside of mitochondria, a situation similar to that in the matrix-ATP-depleted mitochondria (Ref. 9; R. A. Stuart, D. M. Cyr and W. Neupert, unpublished). Taken together, these results indicate an absolute requirement for $\mathrm{mt}-\mathrm{Hsp} 70$ for passage of proteins across the mitochondrial IM.

\section{The role of cytosolic chaperones in mitoshondslal protein import}

It is generally agreed that precursor proteins cannot traverse membranes in a folded conformation. This assumption is based on several observations, in particular: (1) when precursors were bound to antibodies or Fab fragments, import was abolished $^{12}$; (2) chimeric preproteins consisting of mitochondrial presequences and mouse cytosolic dihydrofolate reductase (DHFR) are not imported if the DHFR domain is stabilized by binding of a ligand ${ }^{13}$; and (3) a series of chimeric proteins, consisting of a mitochondrial presequence fused to increasingly large segments of mature proteins containing DHFR at the carboxyl terminus, stall with the folded DHFR domain on the surface of the OM and the amino terminus in the matrix. These fusion proteins have been used to map the minimum polypeptide length required to span the outer and inner membranes, a distance of $-10-12 \mathrm{~nm}$. Roughly 50 amino acids are required to span both membranes $^{14}$. Taken together, these data would suggest that a precursor protein passes through the mitochondrial import channels as an extended polypeptide chain $n^{4,14}$.

At this stage, one might ask what the conformation of a mitochondrial preprotein in the cytosol is. The following findings are relevant in this respect. (1) Preproteins in the cytosol are not usually tightly folded, as they are extremely sensitive to digestion by added proteases. (2) However; domains of some preproteins, such as the DHFR fusion proteins discussed above, can be correctly folded, and these preproteins are still imported efficiently. (3) The presence of a correctly folded domain may, however, at least in certain cases, present a problem for the efficiency of import. Interestingly, fusion proteins carrying mutations in

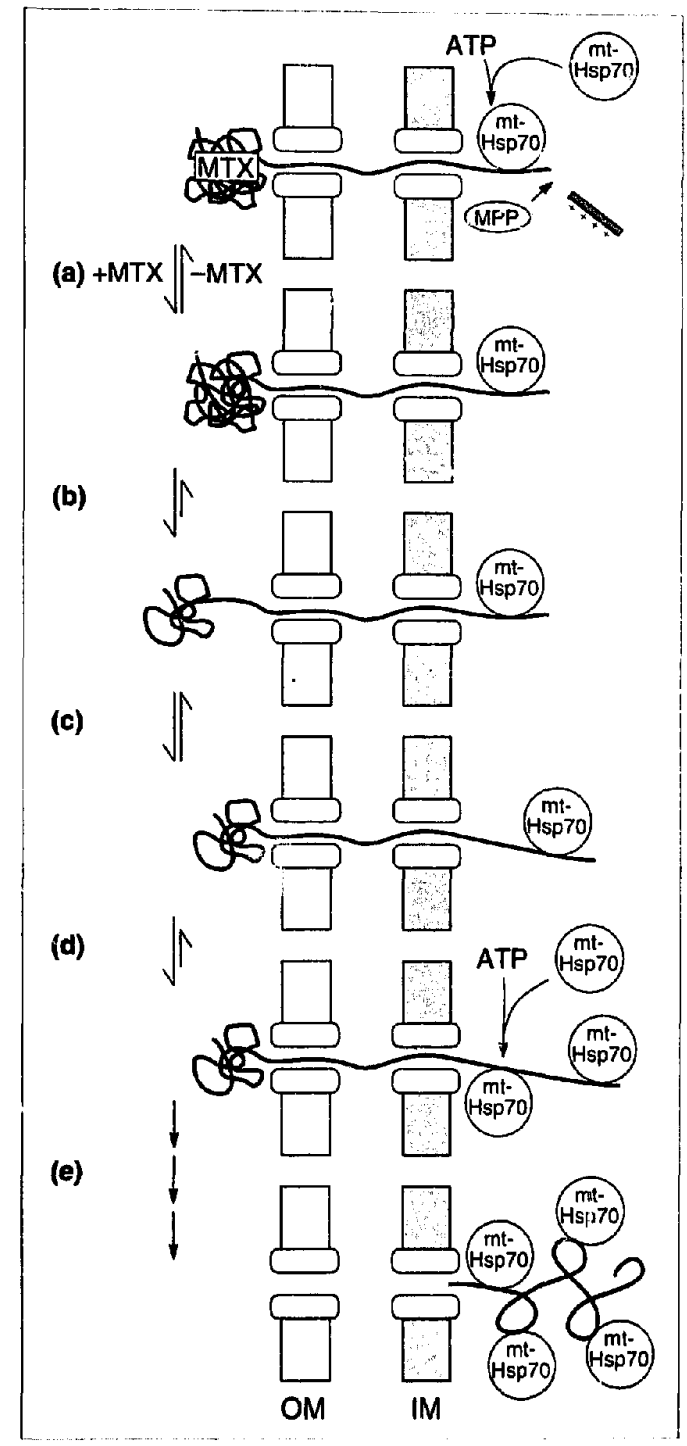

Figure 2

Model of mt-Hsp70-mediated unfolding of preproteins outside the mito-hondrion during the translocation process. Unfolding and translucation of preproteins into the mitochondrial matrix is proposed to occur by the following series of events, using a precursor protein containing a DHFR domain as an example. (a) Reversible stabilization of DHFR by methotrexate (MTX) binding. (b) Removal of MTX leads to spontaneous partial unfoiding (breathing) of DHFR outside the mitochondrion. (c) Unfolded segments of precursor slide in the translocation channel. (d) The ATP. dependent binding of $\mathrm{mt}-\mathrm{Hsp} 70$ to a matrix-exposed segment of the incoming polypeptide serves to secure it inside the mitochondrion, thereby shiting the equilibrium of distribution of the polypeptide. (e) Serial binding (and release) of $\mathrm{mt}-\mathrm{Hsp} 70$ leads to completion of translocation. Abbreviations are as for Fig. 1. 


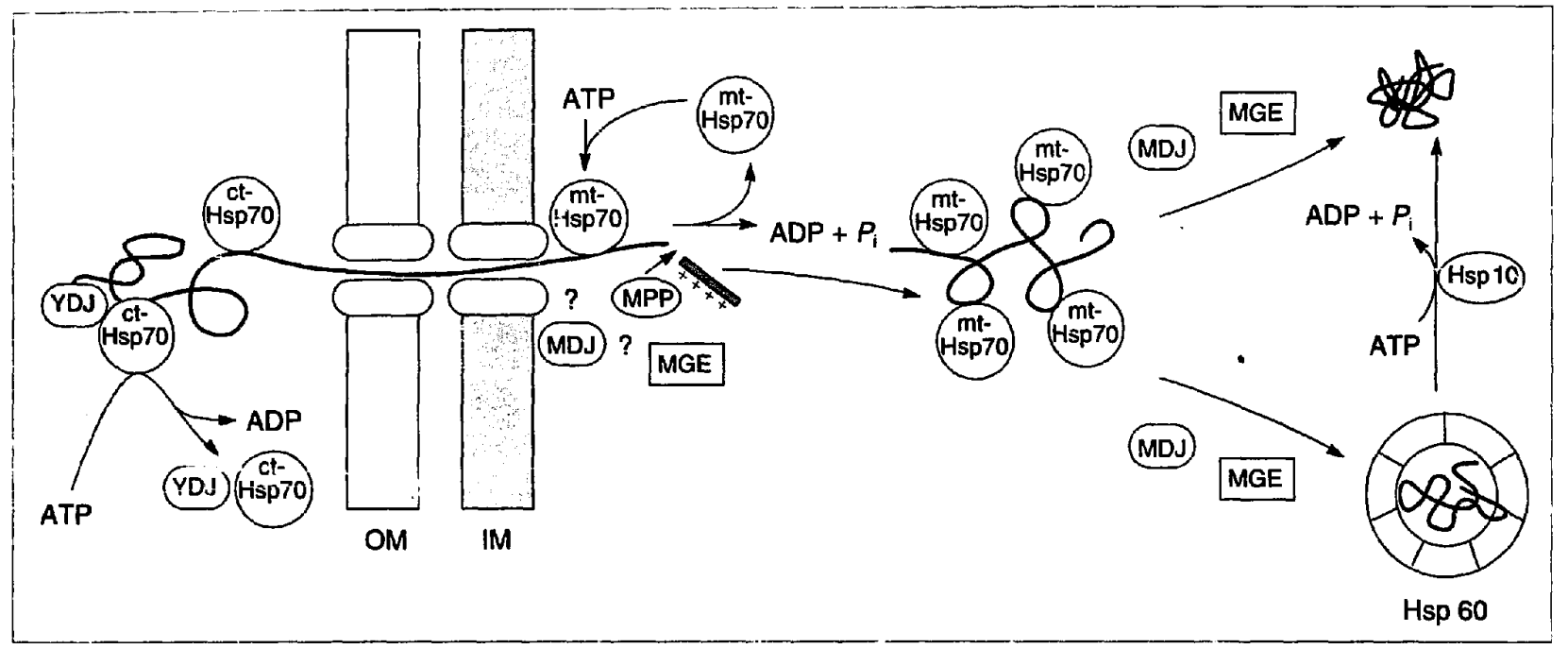

Figure 3

Hypothetical model for the sequential action of mitochondrial chaperorie proteins in the translocation and folding of mitochondrial-matrix-destined precurscr proteins. Cytosolic Hsp70 (ct-Hsp70), together with YDJ, has been suggested to maintain some precursors in an unfolded, translocation-competent conformation befcre membrane trars socation. import of precuronrs into the matrix is mediated by mt-Hsp70 in an ATP-dependent manner. The involvement of MDJ and MGE is presentiy under investigation (see text for detalis). Followinis translocation, precursors are folded directly or become transferred from $\mathrm{mt}-\mathrm{Hsp} 70$ to $\mathrm{Hsp} 60$. Both processes are proposed to require MDJ and MGE. Folding by Hsp60 requires both ATP hydrolysis and the action of a further chaperune, Hsp10. Other abbreviations are as for Fig. 1.

either from a native folded state or from a more loosely folded state. In some instances, it may be necessary to prevent folding of precursors, since unfolding cannot be managed by the transport system. Also, in many cases, it may be necessary to prevent aggregation of precursors, since loosely folded regions have a tendency to aggregate, which may abrogate the ability to become unfolded. How does a cell deal with all these problems? In particular, what is the mechanism that leads to unfolding during import?

Cytosolic chaperones, especially of the Hsp70 family (ct-Hsp70; the SSA gene family in $S$. cerevisiae) have been postulated to interact with precursors in the cytosol. These chaperones, by interacting with precursors, most likely prevent them from folding into a conlormation that cannot be unfolded, and also prevent aggregation ${ }^{16}$. Depletion of ct-Hsp70s in vivo resulted in accumulation of precursors of the $\beta$-subunit of the mitochondrial protein $F_{1}$-ATPase . in addition to members of the Hsp70 family, another chaperone, YDJl, which is a cytosolic homologue of the bacterial Dnal, has been implicated in playing a role in efficient post-translational import into mitochondria, especially at elevated temperatures ${ }^{17-19}$ (the role of this chaperone will be discussed in detail in another article in this series, by D. M. Cyr and M. C. Douglas). The presence of an unfoldase activity, either in the cytosol or on the mitochondrial surface, that would actively support the unfolding of precursors, has not been demonstrated.

\section{ATP-mt-Hsp70 promotes unfolding of precursors outside mitochondrla}

Inhibition of precursor import into mitochondria of $\mathrm{ssc} / 2$ mutanits could be overcome if the precursor was (artificially) unfoided in $8 \mathrm{M}$ urea before import $^{3}$. This suggested that the translocation of an unfolded protein could be driven by this defective mtHsp70, whereas translocation of a partially folded protein could not ${ }^{3}$. Further observations supporting a role of mtHsp70 in precursor unfolding come from studies of the import pathway of cytochrome $b_{2}$ (Refs 7,8 ). Cytochrome $b_{2}$ is targetted to its functional location, the intermembrane space, by a blpartite signal sequence. How this signal operates is a matter of debate, and two models are currently veing discussed, the 'conservative scrilig' and the 'stoptransfer' models ${ }^{20.2 i}$. I i espective of the sorting pathway used, a recent study of the energetics of import has demonstrated a role for $\mathrm{mt}-\mathrm{Hsp} 70$ in unfolaing tightly folded segments of precursor proteins outside the mitochondria. The import of cytochrome $b_{2}$ displayed a very strong requirementit tor inatrix ATP. In the absence of matrix ATP, the pre- cursor accumulated on the outer surface of mitochondria as an unprocessed species that was accessible to externally added proteases. This necessity for matrix ATP reflected dependence on the action of mt-Hsp70, as very similar results were observed in the abovementioned $\mathrm{sscl}-2$ and $\mathrm{sscl}-3$ mutants (Ref. 22; R. A. Stuart and W. Neupert, unpublished). If, however, the precursors of cytochrome $b_{2}$ and derived fusion proteins, which also displayed strong mt-Hsp70 requirements were denatured in $8 \mathrm{~m}$ urea before import, they could be very efficiently imported into both ATP-depleted mitochondria and ssc $1-3$ mutant mitochondria ${ }^{8,22}$.

Cytochrome $b_{2}$ (L-lactate dehydrogenase) contains both flavin and haem as prosthetic groups. About the tirst 100 residues of the mature polypeptide chain form a tightly folded structure which forms the cytochrome $b_{5}$ - or haem-binding domain ${ }^{23}$. This folded domain most probably prevents the stable insertion of the presequence in the matrix, unless unfolded through the action of mt-Hsp70 (see below) . Hence, precursors containing this domain require ATP-dependent binding of mtHspio to the matrix-exposed part of the protein to mediate its unfolding. By contrast precyiochrome $b_{2}$ constructs in which this domain has been deleted or disrupted can be processed with very high efficiency in the apparent absence 
of mt-Hsp70 activity ${ }^{7,8}$. Taken together, the data suggest a function of $\mathrm{mt}-\mathrm{Hsp} 70$ in supporting the unfolding of precursors on the mitochondrial surface. A possible mechanism is that, by binding to segments of precursors on the matrix side, stabilization of an unfolded conformation on the mitochondrial surface is favoured.

\section{Model for the action of Hsp70 in the translocation of proteins into mitochondria}

To summarize, we propose the foilowing model for the translocation of polypeptides into the matrix and the involvement of $\mathrm{mt}-\mathrm{Hsp} 70$. Mt-Hsp70 appears to participate in at least three distinct stages of import which, together, serve to confer unidirectionality and provide the driving force for the import process.

The transport apparatus of the OM guides the amino-terminal presequence of the precursor into the intermembrane space. The translocation machinery of both the OM and the IM is proposed to form proteinaceous channels that mediate the passage of an unfolded polypeptide chain. In response to $\Delta \Psi$, the matrix-targetting presequence of preproteins crosses the $\mathrm{IM}$ in a reversible manner. Binding of $\mathrm{mt}-\mathrm{Hsp} 70$ to the incoming polypeptide upon exposure to the matrix serves to anchor this motif in the matrix and, by so doing, prevents the escape of the precursor back to the cis side of the IM. In this manner, the translocation of the preprotein into the matrix is committed, and mit-Hsp70 has thus conferred unidirecilonality on the process (Fig. 1).

Spuntaneous relaxing, or 'breathing', of the remaining mature parts of the polypeptide chain outside the mitochondrion would then render segments of the protein free to pass across the membrane. In this manner, the protein is translocated in a stepwise manner where further binding of mt-Hsp70 commits increasing amounts of the protein to the matrix and thus shifts the equilibrium of the protein structure outside the mitochondrion towards the unfolded state, which ultimately draws it towards the unfolded preprotein on the trans-side of the IM. If, however, unfolding of domains outside the mitochondria is drastically reduced, for example, by stabilization of a doniain through its interaction with a ligand, there would be no spontaneous sliding across the translocation channel, and mt-Hsp70 could not drive the equilibrium towards the matrix (Fig. 2). This sequence of

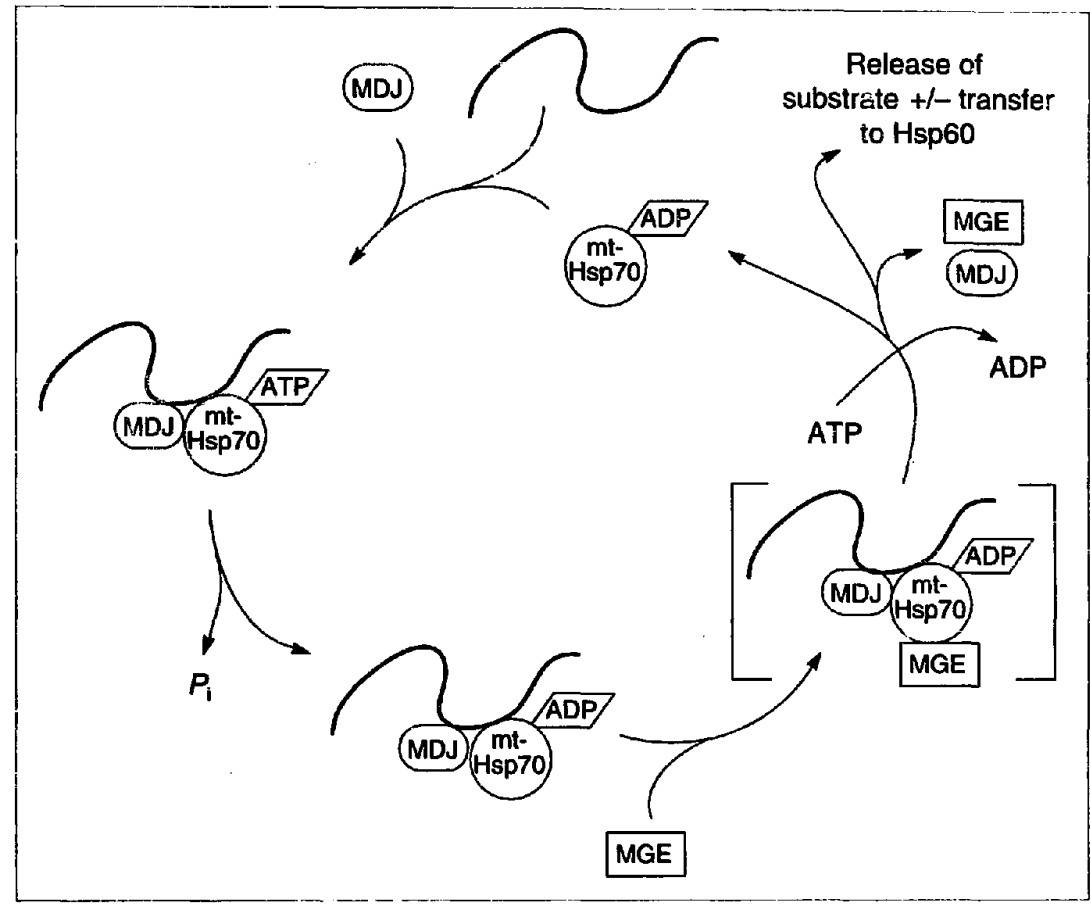

Figure 4

Hypothetical model for the nucleotide-modulated binding and dissociation of unfolded proteins to mt-Hsp70, involving MDJ and MGE. Mit-fisp70-ATP and MDJ are thought to interact with the unfolded newly imported polypeptide, to form an MDJ-mt-Hsp70-ATP substrate complex. Substrate-stimulated ATP hydrolysis converts mt-Hsp70 to a form with high affinity for substrate, mt-Hsp70-ADP. Dissociation of the substrate occurs following the interaction of MGE with this complex. Existence of a stable DnaK-DnaJ-GrpE-substrate complex has not been demonstrated in bacteria, so the analogous mitochondrial complex is depicted here in parentheses. MGE might function by exchanging the bound ADP froin ritHsp70 for ATP. (It is also possible, however, that a nucleotide-free intermediate exists.) Exchange/release of the ADP from mt-Hsp70 results in dissociation of the complex, setting free the substrate and allowing its subsequent transfer to Hsp60 for folding.

events would share a number of features with the 'Brownian ratchet' model proposed recently 24 .

\section{Folding of imported polypeptide chains is mediated by chaperones in the matrix}

Following membrane translocation, newly imported polypeptides have to attain their native conformation in order to function. In many cases, this appears to be ail assisted process, involving the transfer of the imported protein from mt-Hsp70 to another matrix-localized molecular chaperone, Hsp60 (Fig. 3) 3,6,25-27. Folding mediated by Hsp6j is ATP dependent and re quires the action of another chaperone, Hspl0 (Ref. 28). Sequential interaction of imported polypeptides with $\mathrm{mt}-\mathrm{Hsp} 70$ and Hsp60 was suggested following the observation, in sscl-2 mitochondria, that urea-denatured precursor proteins containing DHFR could be imported into the mitochondrial matrix; however, it remained bound to
mt-Hsp70 in an unfolded and proteasesensitive state. This proposal was further supported in another study, where it was shown that a newly imported protein could be successively co-inmunoprecipitated with mt-Hsp70 and Hsp60 (Ref. 6). The details of how a mt-Hsp70-hound imported polypeptide is transferred to Hsp60 are unclear. An analogy can be drawn with the situation in bacteria, where work with reconstituted systems suggested that transfer from DnaK to GroEL is tightly regulated by DnaJ and GrpE29. However, some proteins might be able to fold without the assistance of $\mathrm{Hsp} 60$ and Hspl0, as indicated in Fig. 3.

Recently, a mitochondrial DnaJ homologue, MDJ (mitochondrial DnaJ, termed MDJl in yeast), was identified, and is associated with the inner face of the IM (N. Rowley et al., unpublished). Deletion of the $M D J 1$ gene was not lethal; however, mdj1 nuil mutant cells could not grow at $37^{\circ} \mathrm{C}$ and were petite 
at all temperatures. Folding of newly imported proteins and stability against heat denaturation were reduced in the mdil null mutant. MDJl is thus not essential for folding and refolding of proteins, but makes these processes more efficient and becomes limiting at higher temperatures (N. Rowley et al., unpublished). MDJ1 therefore re seintiles bacterial DnaJ, which is not essential at normal temperatures but is required for refolding of denatured proteins at elevated temperatures ${ }^{29,30}$. Furthermore, a GrpE homologue has been found in yeast mitochondria. Disruption of the gene encoding the mitochondrial GrpE (MGE, mitochondrial $\mathrm{G} \cdot \mathrm{pE}$ ) is lethal in yeast ( $\mathrm{S}$. Laloraya, B. D. Gambill and E. A. Craig, unpublished). The precise functions of MDJ anc MGE are not known. On the basis of the data obtained so far, and of the apparent similarities to the bacterial system $^{31-33}$, a model can be proposed for the cooperation of mt-Hsp70, MDJ and MGE in facilitatung folding and refolding of imported proteins (Fig. 4). This model emphasizes the importance of matrix ATP, which is required to confer binding competence to mt-Hisp70.

The question remains as to whether MDJ and MGE are also required for facilitating import of precursor proteins. With $m d j l$ mutaints, no deficiency in import at normal or elevated temperature was observed, indicating that this chaperone is not necessary for import. Hovever, it cannot be excluded that, under certain condilions, MDJ increases the efficiency of import. Mt-Hsp70 seems to bind to incoming precursor proteins in the absence of MDJ1, and perhaps also to release precursors efficiently. The possible function of MGE in this process awaits further characterization o: the protein.

\section{Concluding remarks}

Analysis of protein translocation into mitochondria has furthered our understanding and appreciation of the importance of molecular chaperones. The participation of these proteins at different stages of miochondrial protein import is vital. Molecular chaperones act in concert to ensure that preproieins to be: translocated can attain, or be maintained in, a conformation compatible with passage across membranes. In addition, their action underlies the driving force (together with $\Delta \Psi$ ) and confers directionality on the translocation event. Can these new insights into chaperone function be rel- evant for other membrane systems? Already the Hsp70 homologue BiP (encoded by $K A R 2$ ) in the ER has been implicated in the passage of proteins across the ER membrane. Interestingly, transport of secretory proteins was defective in kar2 mutants, and preproteins accumulated on the surface of the ER as unprocessed species ${ }^{34}$. It remains to be seen whether similar chaperoneassisted mechanisms also facilitate the translocation of preproteins into chloroplasts and peroxisomes.

\section{Acknowledgements}

We wish to thank 'Thomas Langer for his support and for many constructive discussions. We are also grateful to those colleagues who have shared their unpublished results. This work was supported by grants from the DFG Schwerjunktprogramm 'Heat shock response', the Sonderforschungsbereich 184, Teilprojekt B2 and the Münchener Medizinische Wochenschrift.

\section{References}

1 Kiebler, M., Becker, K., Pfanner, N. and Neupert, W. (1993) J. Memb. Biol. 135 $191 \cdot 207$

2 C. aig, E. A. et al, (1989) Mol. Cell. Biol, 9, 3000-3008

3 Kang, P-J. et al. (1990) Nature 348, 137-142

4 Neupert, W. Hartl, F.U., Craig, E. A. and Pfanner, N. (1990) Cell 63, 447-450

5 Hwang, 5. and Schatz, G. (1989) Proc. NatI Acad. Sci. USA 86, 8432-8436

6 Manning.Krieg, U., Scherer, P. and Schatz, G. (1991) EMBO J. 10, 3273-3280

7 Glick, B. S., Wachter, C., Reid, G. A. and Schatz, G. (1993) Prot. Scl. 2, 1901-1917

8 Stuart, R. A. et al. Eur. J. Biochem. (in press)

9 Gambill, B. D. et al. (1993) J. Cell Biol. 123,
119-126

10 Cyr, D. M., Stuart, R. A. and Neupert, W. (1993) J. Biol. Chem. 268, 23751-23754

11 Scherer, P. E. et al. (1990) EMBO J. 9 , 4315-4322

12 Schleyer, M. and Neupert, W. (1985) Cell 43 330-350

13 Eilers, M. and Schatz, G. (1986) Nature 322 228-232

14 Rassow, J. et al. (1990) FEBS Lett. 275, 190-194

15 Vestweber, D. and Schatz, G. (1988) EMBO J. 7, 1147-1151

16 Deshaies, R. et al. (1988) Nature 332 800-805

17 Caplan, A. J. and Douglas, M. G. (1991) J. Cell Biol. 114, 609-621

18 Atencio, D. P. and Yaffe, M. P. (1992) Mol. Cell. Biol. 12, 283 291

19 Caplan, A., Cyr, D. M. and Douglas, M. G. (1992) Cell 71, 1143-1155

20 Hartl, F-U. and Neupert, W. (1990) Science 247, 930-938

21 Glick, B. S. Beasley, E. M. and Schatz, G. (1993) Trends Biochem. Sci. 17, 453-459

22 Voos, W. et al. (1993) J. Cell Biol. 123. 109-118

23 Xia, Z. and Matthews, F. S. (1990) J. Mol. Biol 212, 837-863

24 Simon, S. M., Perkin, C. S. and Oster, G. F. (1992) Proc. Natl Acad. Sci. USA 89 3770-3774

25 Cheng, M. Y. et al. (1989) Nature 337 620-625

26 Johnson, R. B., Fearon, K., Mason, T. and Jindal, S. (1989) Gene 84, 295-302

27 Ostermann, J., Horwich, A., Neupert, W. and Hartl, F-U. (1989) Nature 341, 125-130

28 Rospert, S. et al. (1993) Proc. Natl Acad. Sci. USA 90, 10967-10971

29 Langer, T. et al. (1992) Nature 356, 683-689

30 Schröder, H., Langer, T., Hartl, F.U. and Bukau, B. (1993) EMBO J. 12, 4137-4144

31 Palleros, C. R. et al. (1993) Nature 365 664-666

32 Llberek, K. and Georgopculos, C. (1993) Proc. Natl Acad. Sci. USA 90, 11019-11023

33 Hartl, F-U., Hlodan, R. and Langer, T. (1994) Trends Biochem. Sci. 19, 20-25

34 Sanders, S. L. et al. (1992) Cell 69. 353-365

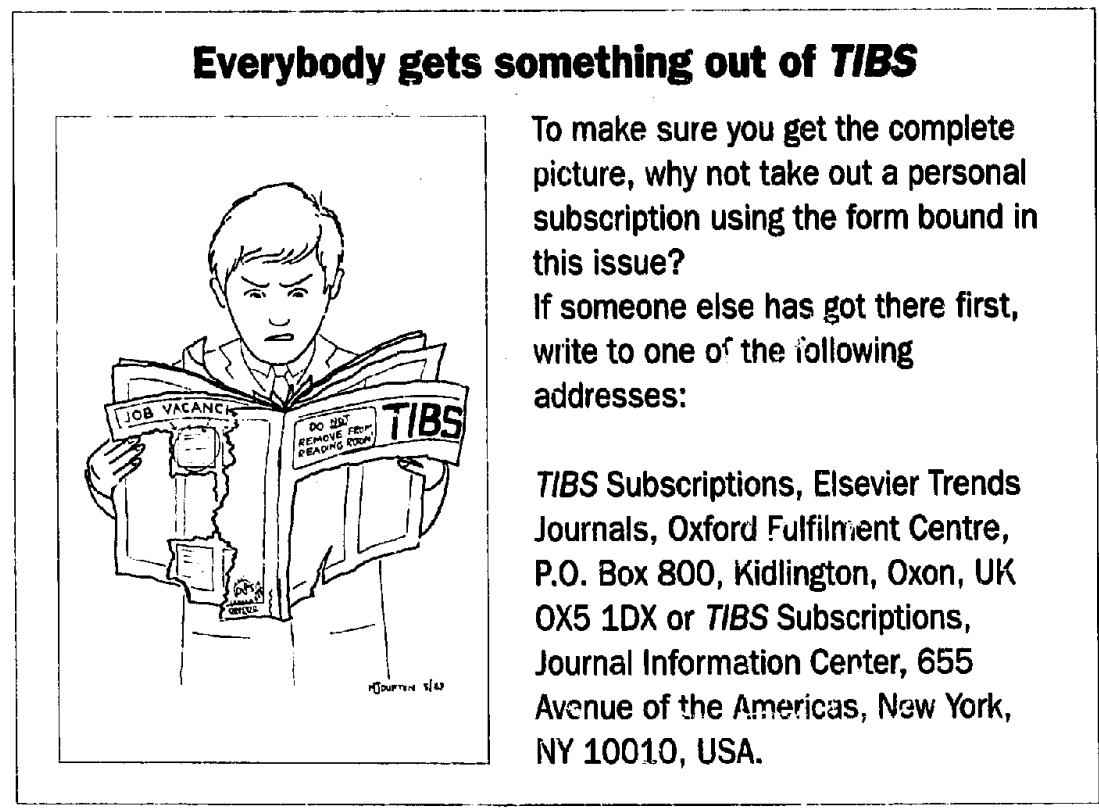

